\title{
Protée
}

\section{Enveloppes, prothèses et empreintes : le corps postmoderne (À propos et à partir de l'étude d'Herman Parret)}

\section{Jacques Fontanille}

Volume 28, numéro 3, 2000

Mélancolie entre les arts

URI : https://id.erudit.org/iderudit/030609ar

DOI : https://doi.org/10.7202/030609ar

Aller au sommaire du numéro

\section{Éditeur(s)}

Département des arts et lettres - Université du Québec à Chicoutimi

ISSN

0300-3523 (imprimé)

1708-2307 (numérique)

Découvrir la revue

Citer cet article

Fontanille, J. (2000). Enveloppes, prothèses et empreintes : le corps postmoderne (À propos et à partir de l'étude d'Herman Parret). Protée, 28(3), 101-111. https://doi.org/10.7202/030609ar
Résumé de l'article

À la lecture du texte d'Herman Parret, consacré à Marcel Duchamp, apparaît de toute évidence qu'une autre sémiose figurative se met en place : là où on attendrait une sémiose (plus ou moins) iconique, dans l'ordre de la représentation des corps (féminins, notamment), Duchamp joue de la contiguïté et de l'empreinte, c'est-à-dire de relations indicielles. Pourtant, l'empreinte est aussi une figure hypoiconique, dans la mesure où elle propose un simulacre et une certaine équivalence d'un corps qui a été en contact avec une forme matérielle, et qui a laissé une trace (une inscription) comme témoignage de ce contact et de son identité en tant que corps. Une sémiotique de l'empreinte se dessine alors, comme une espèce particulière de la mémoire du discours, la mémoire propre à la syntaxe figurative: dès lors que les figures du monde naturel sont considérées comme des " corps », leurs interactions sont inscrites sur leurs enveloppes respectives, et ces inscriptions sont ensuite interprétables comme des figures de discours. Chez Duchamp, un des résultats les plus remarquables du dispositif de l'empreinte est de faire du masculin le moulage ou le complément figuratif, accidentel et contingent, de l'empreinte du féminin. 


\title{
Enveloppes, prothèses et empreintes: LE CORPS POSTMODERNE
}

\author{
(À propos et à partir de l'étude d'H erman Parret)
}

JACQUES FONTANILLE

\section{Introduction}

Sous des apparences de glorification du corps, la civilisation contemporaine en donne pourtant de bien Curieuses représentations.

La tradition phénoménologique, sous le thème de la chair et sous celui du corps propre, a fait du corps le véhiculeprincipal del'intentionnalité, le lieu et lemoyen de notre «être au monde» signifiant. D ans cette perspective, toutes les propriétés associées directement ou indirectement au mouvement sont concernées au premier chef: le corps intentionnel est un corps kinésique et kinesthésique.

La psychanalyse, en revanche, en partant dela notion de «barrières de contact» développée par Freud, a élaboré une théorie des «membranes» psychiques, enveloppes de protection, detri et d'échange, susceptibles de recevoir les empreintes de «signifiants» révélateurs des événements et des structures psychiques. À cet égard, le corps transformé en «M oi-peau » est un corps cœnesthésique, une enveloppe susceptible de fonctionner comme «surface d'inscription», et d'engendrer par débrayage l'ensemble des supports sémiotiques, les substrats matériels du «plan de l'expression».

$M$ aisla sémiotiquen'est pas en reste, puisqu'U mberto Eco, aux chapitres «Prothèses» et «M iroirs» deson Kant et l'ornithorynque ${ }^{1}$, n'envisage dedonner un statut sémiotique aux outils, aux machines et à l'ensemble de la technologied'aujourd'hui que dans leur rapport au corps. À partir du miroir, considéré comme le signe ostensif par excellence du corps, il envisage par exemple le fonctionnement des écrans qui nous entourent comme une démultiplication et une dérive infinie de cette première ostension. D e la même manière, à partir de la prothèse, et de ses propriétés de substitution ou de renforcement du pouvoir-faire et du savoir-faire, et grâce à un débrayage et une dérive progressive, il met en perspective la définition sémiotique des outils, puis des machines.

On pourrait tout aussi bien, en croisant la problématique de la «surface d'inscription » chère à Anzieu, avec celle des prothèses chères à $E c 0$, mettre en perspectivela définition des «interfaces» et del'«ergonomie» à partir des empreintes que laissent les corps dans leur environnement.

Par une étonnante révolution, lemonde des objets et des machines, tout comme celui de l'intentionnalité et de la signification au plan phénoménal, est alors interprétable comme un monde peuplé de corps et de simulacres et de projections du corps sensible du sujet humain. À défaut de s'approprier et de maîtriser concrètement la technique et ses machines, l'individu humain postmoderne s'efforce donc de les penser «à son image», et d'en faire un prolongement sémiotique de son propre corps.

Pour un sémioticien, cette petite révolution épistémologique doit être observée soigneusement, car elle implique deux «conversions» très particulières, qui ont des conséquences théoriques et méthodologiques, peut-être même philosophiques, considérables.

La première de ces conversions réside dans la transformation de la chose, qui a un statut de réalité mais pas de 
propriétés sémiotiques, en un objet qui, lui, est doté de propriétés sémiotiques. Plus précisément encore, la conversion de la chose en objet revient, sur un principe depuis longtemps scruté par $\mathrm{H}$ usserl, à reconnaître dansla chose, grâceà ce qu'il appelleune «saisieanalogisante», des propriétés et un mode d'existence qui sont ceux-mêmes du corps sensible du sujet (de sa chair, dit-il); en bref, il s'agit de faire de la choseun actant sémi otiqueà part entière, sur le modèle de l'actant-corps du sujet.

La deuxième de ces conversions a trait au mode sémiotiquequi permet de dériver lestatut sémiotique des objets, des outils, des prothèses, des empreintes et des machines, à partir decemême corps sensible. La tradition phénomé nologique et toute une partie de la psychologie cognitive nous ont habitués à penser cette conversion comme reposant sur des analogies et un principe de «symbolisation »: le corps, dit en substance M erleau-Ponty, est cet instrument merveilleux dont toutes les parties sont susceptibles de fournir un analogon des choses du monde et qui, par métaphore, analogie ou symbolisme, peut ainsi leur donner du sens.

$M$ ais on voit bien que le principe du «débrayage» et de la dérive définitionnelle, que ce soit chez Anzieu ou chez Eco, qui nous fait passer progressivement de l'empreinte et de la prothèse à toutes les machines, les écrans et les surfaces d'inscription, même si elle suppose au moins un principe de conservation globalement «hypoiconique», n'en use pas moins systématiquement de la contiguïté, de la connexion, de l'homologie, voire de la métonymie.

La «petite révolution» du corps postmoderne - et le mystère de l' «incarnation » du monde des objets- serait donc indicielle plutôt qu'iconique.

C es particularités sont spécial ement mises en évidence dans l'étude d'H erman Parret consacrée à la peinture de $M$ arcel D uchamp.

N ous faisons I'hypothèse, en partant des analyses de Didier Anzieu consacrées aux «enveloppes psychiques»? que l'enveloppe corporelle peut être convertie, sous certaines conditions de débrayage, en une surface d'inscription, pour un mode sémiotique autre et qui, à tout le moins, ne serait déjà plus immédiatement corporel. C'est ainsi que, suivant partiellement en cela Didier Anzieu, nous pourrions considérer la page manuscrite ou imprimée et l'écran de cinéma comme des fragments de la «peau » des choses et du monde des choses, sur lesquels pourraient s'inscrire des énoncés verbaux ou visuels, et qui devraient leur statut de «support sémiotique» au débrayage de l'enveloppe corporelle «multisensorielle».

$M$ ais il faut observer, et même, si possible, prouver l'existence d'un tel «débrayage». Cette démarche est nécessai re, en effet, si l'on veut que lelien entrel'enveloppe corporelle et la surface d'inscription sémiotique ne reste pas unehypothèsepurement spéculative, tout justebonne à satisfaire les penchants psychanalysants d'une certaine génération de sémioticiens.

L'objet de cette étude est donc de prêter attention à certaines des figures de ce débrayage, notamment à celles qui offrent une représentation explicite d'une des formes intermédiaires, entre l'enveloppe proprement dite et la surface d'inscription; on chercherait ici, en quelque sorte, à saisir quelques instantanés dans le processus de débrayage postulé, quelques «arrêts sur image» qui nous convaincraient mieux de l'existence d'une relation et d'une opération orientée entre l'enveloppe et la surface d'inscription.

Ce faisant, il faut garder à l'esprit que, puisqu'il s'agit du débrayage d'un «support» du plan de l'expression, et plus généralement d'une des manières de rendre compte de l'actualisation d'une modalité sémi otique à partir d'un substrat matériel, l'examen des figures en question devra porter tout particulièrement sur leur rôle dans la mise en place (et dans la spécification) d'unefonction sémiotique. $\mathrm{N}$ ous retrouverons alors, pour des rai sons de fond qui ont trait à l'objectif même dece débrayage, la distinction entre les types de sémiosis: par analogie, par contiguïté, etc.

En raisonnant simplement à partir des phénomènes qui sont en cause, à savoir d'un côté un corps (et sa structure sémiotique), et de l'autre des objets, et lemonde des objets, en attente d'une organisation signifiante, on voit tout de suite à quelles sortes de figures il faudrait faire appel pour dévoiler le processus de ce débrayage postulé, quelles sortes de figures pourraient constituer de bonnes candidates au titre d' «intermédiaire» (sinon, même, d'«interface») entre lecorps et les objets: on pense ici aux prothèses et aux outils qui, sans être des parties du corps, se substituent à elles, les prolongent, les complètent ou leur procurent des fonctions nouvelles et 
qui, sans être vraiment des objets à part entière, en possèdent pourtant les principales propriétés, et notamment unecertaineautonomie technique, modaleet morphologique.

Parmi toutes les capacités de ces objets, nous nous intéresserons plus particulièrement à leur capacitéà fonctionner eux-mêmes comme surface d'inscription. Si on pense, par exemple, aux effets visibles del'ergonomie sur les outils, on est bien obligé de constater que, au moins pour les parties de l'outil qui doivent être en relation avec I'opérateur humain (parties que I'on pourrait regrouper sous l'intitulé générique «interface sujet»3), elles présentent comme «en creux» et en miroir la forme et les propriétés des parties de l'enveloppe corporelle dont elles accueilleront le contact: des manches d'outils creusés par des doigts absents, des sièges-autos qui épousent des arrière-trains fictifs, des machines à café qui offrent leurs tableaux de comman des à des mains habituées à pian oter sur des claviers, etc.

D ans le mouvement même du débrayage qui, partant d'un corps, engendre des objets signifiants pour cecorps, I'objet (l'outil) gardela mémoire deson originecorporelle, en même temps qu'il anticipe sur son retour au corps. II garde l'empreinte de l'enveloppe corporelle, il en est le signe, au moinslesignede son statut d' «objet-susceptiblede-se-substituer-à-une-partie-du-corps», del'accueillir ou de la compléter. L'empreinte sera donc une des figures qui, comme dans un «arrêt» sur image, nous permettra de comprendre la nature et les enjeux du débrayage de la surface d'inscription 4 .

\section{D igression apéritive: \\ l'art conceptuel comme sémiosis}

M arcel D uchamp, commele rappelle H erman Parret, est surtout connu pour ses provocations à l'égard de la conception traditionnelle (et mêmemoderne) del'œuvre d'art et de la fonction del'artiste, et plus précisément par le déplacement bien connu qu'il opère, en faisant porter tout le poids axiologique de l'art (la «val eur » de l'œuvre en tant qu'œuvre d'art) sur l'acte artistique, et non sur son produit, l'œuvre; I'artiste, en choisissant, assemblant ou construisant des objets, les constitue du même coup en objets artistiques, parce qu'il se pose lui-même, par son geste, comme artiste.
Le ready-made est l'expression la plus connue de ce déplacement, qu'on qualifie de «conceptuel», mais qui, à l'analyse, semble surtout «performatif». Certes, l'égouttoir à bouteilles tout comme l'urinoir n'accèdent au statut d'œuvre d'art qu'en tant que «concepts», puisqu'il faut bien les distinguer des «occurrences» triviales qui leur servent de support. $M$ ais le faireartistique, en quelque sorte épuré de toute exigence de création au sens traditionnel, de création d'objets qui seraient à la fois différents des objets du monde naturel et étrangers au quotidien, en est réduit à se poser lui-même, performativement, comme porteur du pouvoir de conversion artistique.

Le terme même de «concept» (il y aurait d'un côté des objets ordinaires et de l'autre des «concepts objets») prête à confusion, puisqu'il supposerait une abstraction général isante, le passage d'une occurrence à un type, une abstraction qui retirerait toute valeur référentielle particulière à l'œuvre d'art. Pourtant, M arcel D uchamp n'a cessé de donner à ses œuvres, jusqu'à la provocation la plus dérisoire, des titres figuratifs, et d'autant plus figuratifs et concrets que l'apparence de l'objet ou celle de la peinture sont abstraites; tout se passe comme si, par une dénégation persistante, il voulait interdire au «concept » de s'abstraire et de quitter l'objet-occurrence où il s'incarne.

En outre, toute démarche généralisante (le chemin qui conduit del'objet au concept en quelque sorte) repose sur des séries d'analogies qui, pas à pas, permettent d'étendre, puis de délimiter le territoire d'une classe d'équivalences. Or, pour la même raison (les titres toujours figuratifs et concrets), mais aussi parce que les objets esthétiques de $D$ uchamp ne cessent jamais de peser leur poids de présence et d'évidence paradoxales, irréductiblement singulières, il ne semble pas que le «concept» puisse, dans son œuvre, être assimilé à de telles classes d'équivalences.

De fait, l'acte artistique, le faire esthétique selon Duchamp, consiste à déceler, dans l'objet même, une autre dimension, une dimension restée cachée jusqu'alors et qui, par le déplacement performatif dont nousfaisions état plus haut, est mise à jour: en chaque objet gît son propre «concept » artistique, qui ne se donne à voir que si le bon «médiateur» accomplit l'acte adéquat. D ès lors, on peut considérer qu'en chaque objet il y a toujours au 
moins deux faces: une face «occurrence matérielle» et uneface «concept artistique», qui seraient commele recto et le verso de la même feuille de papier, pour reprendre l'image chèreà Saussure. C es deux faces seraient à la fois indissociables, comme le recto et le verso de la feuille, et pourtant on ne pourrait jamais, sans une importante déformation del'objet, les saisir ensemble et d'un même point de vue.

Cette proposition conduit d'emblée à une spécification dela «méta-sémiotiqueimmanente» qui s'exprime dansl'œuvre de D uchamp: on pourrait, sans imprudence excessive, considérer que les deux faces de l'objet, sa face d'occurrencematérielleet sa face de concept artistique, sont les deux plans d'un langage, et donc réunis par une fonction sémiotique; par convention et pour simplifier l'exposé, nous appellerons le premier la chose et le second I'objet, la chose en tant qu'occurrence matérielle et l'objet en tant que concept artistique.

$M$ ais la relation entre les deux plans de ce langage artistique est bien particulière. D'abord, la relation de nécessité (la présupposition réciproque) qui unit les deux faces, la chose et l'objet, repose sur une contiguïté parfaite: si on peut passer ainsi de l'une à l'autre, c'est justement parce qu'il n'y aucun hiatus entre les deux faces, même pas, commedans l'exemple de Saussure, l'épai sseur d'une feuille de papier.

M aisc'est aussi, du même coup, la raison pour laquelle on ne peut les saisir ensemble: comme rien ne les sépare, on ne peut êtreque dans le point de vue del'une ou dans le point de vue de l'autre, et chacun des deux points de vue, chacune des deux faces, contient l'autre en entier, mais virtuellement.

La deuxième propriété de la relation sémiotique entre la choseet I'objet tient donc dans cebasculement des modes d'existence: aussi longtemps que I'un des deux est actualisé ou réal isé, l'autre est virtualisé ou potentialisé, et réciproquement. Autant direqu'il s'agit d'unefonction sémiotique qui n'est saisissable que dans le temps, grâce à la mémoire qui, par exemple, quand on saisit l'objet conceptuel, maintient la présence rémanente de la chose matérielle.

Le recto et le verso de la feuille de papier sont unis, justement, par la feuille depapier, c'est-à-dire par la figure elle-même; si nous poussions un peu plus loin l'analogie avec la sémiosis artistique «conceptuelle» de D uchamp, et si le recto correspondait à la chose matérielleet le verso à I'objet conceptuel, alors on pourrait dire que, lors du passage de la chose à l'objet, lors de la conversion temporelle des modes d'existence, le principe d'identité est respecté, la permanence de l'«entité» soumise à ce traitement artistique est assurée par la figure. La figure serait à cet égard le principe de stabilité et de continuité qui, au moment même où on change de perspective, fait perdurer la précédente et autorise ainsi la production d'une relation sémiotique; mais, au moment même où elle assure la continuité et la permanence entre la chose et l'objet, la figure les met en concurrence et en tension: en ce sens, elle participe de la dimension rhétorique (tensive) du discours artistique. Si même on considère, commeJ ean-F rançois Bordron, que la stabilité figurative est la propriété définitoire même de l'icône ${ }^{5}$, alors on peut préciser et dire que la chose et l'objet se conservent I'une dans l'autre et réciproquement, grâce à l'i cône.

Ainsi en va-t-il, du moins, pour le ready-made. $M$ ais I'œuvre de Duchamp ne se limite pas à cette pratique conceptuelle «alternative»: ou l'une, ou l'autre, des deux faces de l'objet.

En reprenant le fil de l'argumentation qui précède, on voit bien que l'obstacle à l'actualisation simultanée des deux faces, la chose et l'objet, tient au fait que leur relation ne peut s'établir que dans le temps et grâceà une mémoire inscrite dans ce qu'on a appelé la figure (ou I'icône). Toute la question se résume maintenant en ce point: existe-t-il des réalisations possibles de la figure qui autoriserai ent une saisiesimultanée de la choseet del'objet? $\mathrm{O} u$, en d'autres termes, est-il possible de mettre en scène spatialement, et non seulement temporellement, la relation de nécessité entre les deux faces du signe artistique conceptuel?

Indépendamment del'art de $M$ arcel D uchamp, nous connaissons déjà un type defiguresémiotiquequi répond à cette attente et qui, en outre, autorise même une dissociation, éventuellement même définitive, entre les deux faces du signe: c'est l'empreinte, et sa version technique et volontaire le moule'

Par définition, dansl'empreinterien ne séparela chose de l'objet, sinon un changement de statut et, surtout, une disjonction spatio-temporelle: quand il s'agit d'un masque funéraire, par exemple, le moule conserve une forme dont la chair a disparu, mais que n'importe quelle 
autre matière peut venir remplacer; quand il s'agit de l'empreinte relevée par l'enquêteur, la figure établit le lien entre la partie du corps de celui qui est passé sur les lieux, et qui est supposé avoir accompli un forfait, et la partie du corps d'un $X$ à identifier, qui habite quelque part, qui a des habitudes, un métier, une famille, etc. $D$ ans le premier cas, il s'agit du même visage, l'un étant de chair, l'autre virtuel ou d'une autre matière; dans le second cas, il s'agit du même pied ou de la même main, mais avec un changement de rôle figuratif.

L'empreinteréal ise toutes les conditions dégagées plus haut à propos del'«art conceptuel »deD uchamp: 1) une contiguïté parfaite, sans solution de continuité, 2) un nécessaire basculement des modes d'existence. La première condition est si bien réalisée que l'empreinte est considérée comme témoignage, preuve, signature individuelle: à cet égard, elle assure une parfaite continuité entre les deux statuts de l' «entité»; la seconde l'est tout autant, car pour que l'empreinte puisse fonctionner en tant que telle, il faut bien que la première «face» disparaisse et que l'autre apparaisse: aussi longtemps que la main reste posée sur la surface où elle appose ses traces, il n'y a pas, à strictement parler, d'empreinte; aussi longtemps que le visage moulé reste dans le moule, il n'y a pas de moulage, mais un simple masque de plâtre ou d'argile. II faut que les deux «faces» soient l'une actuelle et l'autre potentielle pour que l'empreinte puisse fonctionner comme «signe», impliquant ainsi des processus interprétatifs et persuasifs, des stratégies de réminiscence et de témoignage, etc. ${ }^{7}$

L'empreinte est donc la figure, stable aussi bien dans l'espace que dans le temps, qui permet d'établir le lien entre deux faces, deux statuts, deux états ou deux rôles dela même «entité» et qui manifesteainsi une desformes possibles de la «mémoire sémiotique».

$G$ reimas insistait, notamment pour justifier la syntaxe du carré sémiotique ${ }^{8}$, sur la mémoi re du di scours: une affirmation qui suit une négation n'a pas la même valeur qu'une affirmation qui en suit une autre. La question de la mémoire du discours est pourtant beaucoup plus générale; on peut même direqu'elle concernetout univers sémiotique, mêmele mondenaturel, pourvu qu'on puisse y reconnaître une syntaxe. C ette mémoire serait, comme dans les systèmes physiques quantiques, la mémoire des interactions entre entités sémiotiques: on admet bien, par exemple, que la rencontre entre un sujet et un anti-sujet est susceptible de les transformer durablement tous les deux, et, par exemple, d'augmenter lepouvoir faire, lesavoir faire ou le vouloir faire du sujet; on admet aussi que la rencontre sensibleet affective entre un sujet et un objet de valeur soit susceptible de transformer durablement ses passions. M aisil faudrait aussi admettre queles interactions entre figures sont aussi susceptibles de les modifier durablement, dans la perspective d'une syntaxe figurative.

$D$ ans tout univers figuratif, et tout particulièrement dans le monde naturel, tout déploiement d'énergie, tout mouvement d'une seule figure est susceptible de modifier durablement l'enveloppe d'une ou plusi eurs autres: c'est l'érosion éolienne des reliefs, c'est la trace de pas sur le sol, c'est l'objet renversé ou brisé par le passage d'un être animé. Cette suggestion ne doit pas surprendre: elle découle tout simplement d'un principe très général, à savoir que toute la syntaxe figurative repose sur l'interaction entre matière et énergie, et que les équilibres stables ou instables de cette interaction produisent des figures identifiables. D ès lors que les objets sont traités comme des corps en interaction, et pas seulement comme des figures abstraites, et pris dans une syntaxe figurative, I'interaction entre matière et énergie se met en scène comme interaction entre mouvements des uns et enveloppes des autres.

N ous appellerons «marquage» ce principe syntaxique général de modification durable des entités sémiotiques par les interactions antérieures: ce principe suppose au moins que ces entités, outre leur rôle purement formel, obéissent à un autre principe, celui d'identitéet de permanence. La chaîne et la syntaxe des «marquages» constituent la «mémoi re» du discours et, à ce titre, elles ne diffèrent pas des autres phénomènes syntaxiques évoqués plus haut (les modifications modales et passionnelles qui affectent les sujets dans leurs parcours). Enfin, dans lecas particulier des entités figuratives, et tout particulièrement desfigures traitées comme des corps, alors les «marquages» sont des «empreintes» et la «mémoire» du discours, constituée dans ce cas particulier du réseau de ces empreintes, formera ce quenous avons appeléjusqu'à présent la «surfaced'inscription ». La surface d'inscription est en somme la mémoi re figuratived'un univers sémiotique, cette enveloppe constituée de la totalité des «souvenirs» destimulations, interactions et tensions reçues par la figure-corps. 
Cet ensemble conceptuel, marquage, mémoire, empreinte, mémoire figurative, surface d'inscription, est à mettre au compte d'une constitution de la syntaxe figurative du discours.

L'empreinte est donc la figure, stable aussi bien dans l'espace que dans le temps, qui permet d'établir le lien entre deux faces, deux statuts, deux états ou deux rôles de la même «entité». 0 n doit donc se demander maintenant si, chez $M$ arcel D uchamp, la figure del'empreinte ou du moulage est susceptible de réunir la «chose matérielle» et son «objet conceptuel ». II semble que cette figure soit systématiquement mise en œuvre dans les représentations du corps, notamment du corps féminin.

\section{À propos de la représentation du corps féminin}

Les critiques et commentateurs de l'œuvre peinte de D uchamp ont tous identifié le rapport très particulier que l'artiste entretenait avec le corps féminin, et notamment sa conception très personnelle de l'érotisme. Citons H erman Parret:

Le corps selon Duchamp ne se laisse prédiquer d'aucune catégorie esthétique - le corps n'est ni beau, ni sublime, ni gracieux, ni dégoûtant non plus-, aucune intériorité ne s'y manifeste, aucune phénoménologie n'y découvrira jamais quelque signifiance. II sagit en fait du corps essentiel, le corps marquépar le sexe et la mort, par Éros et Thanatos, et par rien d'autre. Q uand Cabanne questionne D uchamp sur le rôle de l'érotisme dans son cauvre, il répond: "Énorme. Visibleou sous jacent, partout», et $D$ uchamp énonceà J ouffroy quelesexeest la seulechosequ'il prend vraiment au sérieux. ${ }^{9}$

Pourtant, la confrontation entre, d'une part, cette affirmation générale et de principe et, d'autre part, la représentation qu'il donne des corps féminins laisse dubitatif: des corps étalés comme des masses de chair inerte et amputés (cf. Étant donnés: $1^{\circ}$ la chute d'eau, $2^{\circ}$ le gaz d'éclairage, 1946-1966), des corps transformés en assemblages de formes hétéroclites: tuyaux, récipients, «machines» faites de volumes comportant des parties renflées et des parties amincies et communiquant entre eux par des tiges ou des tubes (Vierge, 1912 [ill. 1]; Le Passage dela Viergeà la M ariée, 1912 [ill. 2]; M arié, 1912 [ill.3]). Ces représentations selai ssent pourtant disjoindre en deux ensembles:
1) d'un côté, les masses charnelles, allongées ou debout, inertes ou vives, dont lechromatismeet lesformes évoquent avec quelque ressemblance ceux des enveloppes corporelles (LeBuisson, 1911; L egaz d'édlai rageet la chute d'eau, étude préliminaire de Étant donnés...,1948-1949 [ill. 14]; Étant donnés: $1^{\circ}$ la chute d'eau, $2^{\circ}$ le gaz d'éclai rage, 1946-1966);

2) de l'autre, les assemblages de volumes et de lignes, tous traités dans des variétés de brun, ocre et rouge (Le Grand Verre, 1915-1923; la Vierge [ill. 1]; Le Passage de la Vierge à la M ariée [ill. 2]; la M ariée, 1912 [ill.3]).

D ans le premier cas, on n'est guèretentéde rechercher quelque stratégie «conceptuelle» que ce soit: malgréleur caractère parfoistourmentéou perturbant, cescorpss'inscrivent dans la tradition du «nu» féminin en peinture. $D$ ans le second cas, on pourrait se contenter d'invoquer l'esthétique cubiste. $\mathrm{M}$ ais, comme le fait remarquer H erman Parret, l'exercice de décomposition cubiste ne s'exerce plus ici sur l'enveloppe corporelle, sur les parties visibles que sont le torse, la tête et les membres, mais sur la composition intérieure du corps:

Le désir d'homme, le regard voyeuriste est invité par D uchamp à di sséquer avec une précision diagrammatique la machine cadaverique de la Femme et il y découvre ses secrets intimes, des organes viscéraux reliés par des tubes, trompes et cylindres. [...] La M ariée du G rand V erre est l'aboutissement par schématisation, par épuration, rien que les organes essentiels pour le fonctionnement essentiel, celui d'aimer pour mourir. 10

La plupart de ces «femmes essentielles» comportent, au centre, une figure récurrente qui devrait en assurer la reconnaissance ou, du moins, fournir un élément de permanence et de cohérence minimales à la représentation du corps: il s'agit d'un volume composéen son centre de deux cônes accolés, formant à eux deux un renflement terminé par deux sommets, et de deux tubes qui prolongent, respectivement vers le haut et vers le bas, les sommets des deux cônes; le tube supérieur, plus long que l'inférieur, est lui-même prolongé par deux tiges parallèles au bout desquelles se tient une forme «en éventail » ou, du moins, en demi-cercle. C e motif, repris trois fois dans le corpus ${ }^{11}$, se présente, comme bien 
d'autres dans la même série, comme un contenant opaque dont on ne connaîtrait jamais le contenu.

Pourtant, ce n'est pas ainsi qu'il convient de voir de telles formes: si on veut bien imaginer qu'au lieu de contenants il s'agit de contenus dont le contenant aurait disparu, on comprend immédiatement que, si cesformes cubistes schématisent quelque chose, c'est bien le système des organes internes, et non le corps visible lui-même. Arrêtons-nous un instant sur cette hypothèse.

Pour voir les organes internes, il faut enlever le «corps» lui-même (sa chair, son squelette) ; les viscères sont, avec le corps visible, dans le même rapport que l'empreinte et I'objet: quand I'un est actuel, l'autre est potentiel; la forme extérieure des viscères est l'empreinte (plus ou moins exacte) de la forme intérieure du corps, forme qui n'est accessible que si ce dernier est absent. M ais cela n'empêche pas que cette forme-empreinte puisse ellemême être le contenant des fluides essentiels de la vie et de la mort.

Le motif «aux deux cônes et aux deux tubes» est parfaitement adaptéà cet usage: formestable, y compris d'un tableau àl'autre, ouvert aux deux bouts, il «communique» en deux dimensions: d'un côté, en tant que forme de l'enveloppeintérieuredu corps absent, il en est néanmoins I'indice permanent; de l'autre, en tant que volume prolongé par deux tubes, il est un lieu de passage et de conversion des «fluides essentiels».

II est vrai que $M$ arcel Duchamp est fasciné par les machines ${ }^{12}$, et qu'elles lui fournissent une grande partie desmotifsici invoqués; maisl'important, en l'occurrence, ne tient pas tant aux motifs qu'à leur syntaxe: que le corps de la femme soit ainsi «machinisé» est certes surprenant, mais la signification de cette machinisation réside dans la figure del' «empreinte». I ndissociablement lié au corps-chair extérieur, un autre corps existe, celui dela vie et dela mort, celui du sexeet desfluides essentiels. Et ces deux corps, parfaitement contigus I'un à l'autre, sans hiatus, ont une limite commune, la «forme-empreinte» qui sera considérée, selon qu'on adopte le point de vue de l'un ou de l'autre corps, comme le moule des viscères ou comme l'enveloppe de la cavité intérieure du corps: du point devuedu «corpsessentiel », la «machine» de vie et de mort, le corps-chair n'est que la matière d'un moule où prennent place les organes vitaux; du point de vue du «corps-chair», les viscères ne sont que le rem- plissage del'enveloppeintérieure. La «figure»delaformeempreinte est donc celle même qui assure la coprésence (en mode virtuel/actuel) des deux faces du corps.

\section{Figures de l'empreinte et du moulage du corps intérieur}

H erman Parret a identifié plusieurs empreintes et moulages de sexes. Par exemple, une très curieuse représentation d'un objet en forme de cylindre-canal, incurvé en son extrémité, au long duquel prennent place deux renflements quasi sphériques (la Valve Auvard [ill.5]): un instrument gynécologique destiné à être inséré dans I'utérus. L'instrument en question correspond donc à l'empreinteinverse, celledela formeintérieure du viscère, mais considérée, si l'on peut dire en ces matières, comme «homothétique» et «isomorphe» de sa forme extérieure. Certes, l'instrument n'a plus le statut exact d'une empreinte, puisqu'il ne sert pas à reconnaître ou pérenniser une forme ${ }^{13}$, mais le dessin qu'en fait Duchamp lui confère celui d'un moulage.

D'autresœuvres (Prièredetoucher, 1947 [ill. 9]; Feuille devignefemelle, 1950 [ill. 10]) se présentent explicitement comme des moulages de sexes ou de seins féminins. U ne autre (Coin dechasteté, 1954 [ill.15]) affiche directement le rapport intimemoule/moulage, grâceà l'emboîtement strict de deux formes de couleurs différentes, sans qu'on puisse identifier la nature des parties en présence, bien que letitre, toutefois, Coin dechasteté, nous inciteà rester toujours sur la mêmeisotopie. La plus curieuse detoutes, dans sa simplicité apparente, est sans doute I' $O$ bjet- $D$ ard (1951) [ill.11]: il semblerait, et le titre encourage à une telle interprétation, que ce soit une représentation phallique; mais elle serait, en tant que telle, bien peu conventionnelle (fortement coudée) et sans grande précision descriptive. 0 n pourrait tout aussi bien fairel'hypothèse que, tout commel'instrument gynécologiquese présentait comme le moulage de la forme intérieure des viscères génitaux, cet $O$ bjet- $D$ ard figurerait le moulage du vagin, et non celui du pénis.

La figure sémiotique del'empreinte acquiert al ors une toute autre dimension : non seulement chaque partie du corps semble pouvoir recevoir le même traitement quele corps tout entier, c'est-à-dire une division en deux faces indissociables et complémentaires, avec une pelliculefron- 
tière qui fait office d'empreinte ou de moulage, mais, en outre, le rapport contenant/contenu se trouve clairement (voire obsessionnellement) thématisé.

II s'agit alors de la différence sexuelle - voire des rapports érotiques- qui investit de son propre contenu sémantique les propriétés formelles de l'empreinte: contiguïté en continuité et basculement des modes d'existence; le féminin et le masculin ne sont que des domaines contigus, supposés se retrouver en contact intime selon le principe de la «vérification des empreintes»: deux faces et deux rôles différents et successifs, mais dont le lien nécessaire (et la fonction sémiotique) est exprimé par la stabilité de leur empreinte commune (enveloppe interne pour la partie féminine, enveloppe externe pour la partie masculine) ${ }^{14}$.

À cet apparent équilibre, il faut cependant apporter deux correctifs. D 'un côté, le «masculin »n'apparât, dans cette affaire, que comme l'éventuelle réalisation, occasionnelle, de la formeintérieure du «féminin »: la preuve en est que cette forme existe en l'absence de toute expression masculine. La figure de l'empreinte, dans la version sémiotique que nous en avons donnée, est claire à cet égard: la seconde face n'a que le second rôle, rôle accessoire de confirmation, et que l'on confronte pour vérification àl'empreinte, dans un processus interprétatif/ persuasif, alors que la première face a le premier rôle, celui qui fait tout le drame et l'énigme del'histoire, celui du protagoniste principal (le criminel, levivant, etc.). Le masculin, en somme, ne serait que l'instrument qui permettrait de faire «fonctionner» l'empreinte du féminin. En termes peirciens, le masculin ne serait qu'un desinterprétantspossibles du signeféminin et de son repre sentamen, la forme-empreinte.

D 'un autre côté, la thématique sexuelle n'est qu'une parmi d'autres. Certes, elle est particulièrement mise en évidencepar $D$ uchamp et par son commentateur, $H$ erman Parret; mais la représentation du corpsféminin en formes de récipients et de tubes, on l'a déjà fait remarquer, contient tous les «fluides essentiels», ceux de la vie et ceux de la mort. De fait, ces fluides sont ceux mêmes qui émanent du monde extérieur et qu'on pourrait désigner, sans a priori sur leurs investissements axiologiques (vie ou mort), comme les «fluides de l'altérité».

Dès lors, la récurrence desempreintes et des moulages intérieurs devient, chez Duchamp, une sorte de mé- ditation sur les relations entre le même et l'autre, sur la capacité du corps féminin à faire, à partir de cette dialectiquepurement phénoménologique, œuvrethématique et axiologique de vieet de mort: à cet égard, I'activitédu corps féminin, considéré comme «concept-corps», consisterait à transformer toute altérité en «mêmeté»; mais le «même», en l'occurrence, n'est pas celui que fondent I'analogie ou l'équivalence, mais très précisément celui qu'authentifient l'empreinte et le moulage. II s'agit sans conteste d'un «même» obtenu par individuation et adé quation, et non par généralisation et constitution de classes d'équivalences.

Si lecorpsféminin est unemachine, c'est unemachine à «s'approprier »|'autre pour en faireun contenu contigu et parfaitement adéquat 15 .

\section{M oulages et empreintes de l'enveloppe extérieure}

Les représentations plus «classiques» des corps pré sentent toutes une caractéristique qui a retenu l'attention d'H erman Parret: une sorte de halo entoure le corps et semblel'isoler du fond. II s'agit plus précisément du dessin de La M ariée mise à nu... (1968) [ill.6], du Portrait du D octeur D umouchel (1910) [ill. 7] et du Buisson. Q ue ce soit une zone blanche comme dans le dessin de La Mariée..., un halo rouge sur fond brun comme dans Portrait du D octeur D umouchel ou un large halo-cocon bleu comme dans Le Buisson, cette zone qui entoure le corps a pour premier effet d'empêcher lefond du tableau d'apparaître comme un fond pour la figure du corps: au contraire, tout se passe commesi la figure qui se détache sur le fond était le halo lui-même, comme une sorte d'objet-moule ou cocon qui contiendrait le corps. De fait, la figure du corps n'a d'autre fond que cette épaisse enveloppe de matière chromatique, sur laquelle elle se détache sans aucune profondeur et qui elle-même se détache comme une figure en relief sur le fond général du tableau.

D ans Portrait du D octeur D umouche [ill. 7], la main du personnage nous fournit en quelque sorte la «clé» de ce dispositif: le halo qui entourela main gauchese donne al ors très clairement à voir comme un moule, sur lequel la main apposée dessine en creux des zones sombres, entourées de zones plus claires. Les règles d'interprétation conventionnelles de la représentation des corps nous 
invitent à les considérer comme des représentations tridimensionnelles en relief: les modelés avancent vers le regard, les creux reculent, etc. $M$ ais la forme de la main peut sans difficulté se lire aussi, à cause du dispositif en «halo », comme une trace laissée en creux dans la matière d'un moule, ou au moins commeunemain en relief dont la pression commence à l'imprimer en creux dans la matière du fond.

Le corps féminin, dans le dessin de La M ariée..., n'est qu'un contour, enveloppé de blanc, et tout aussi blanc à l'intérieur: une trace, une inscription dans la matière d'une surface évidée et décolorée. Les deux femmes dans le paysage Le Buisson, chair pleine et robuste, résistent mieux à cette lecture inversée; pourtant, elles aussi, globalement, imposent une sorte d'affaissement enveloppant à cette zone qui les entoure: même s'il est plus difficile de voir les modelés de leurs corps comme des creux imprimés dans lazone bleue, on nepeut pas ignorer le léger enfoncement des deux figures à l'intérieur du «halo», qui forme comme une sorte de «coque» pour mieux les accueillir.

Globalement, le rapport moule/moulage, matière/ empreinte se substitue donc au rapport figure/fond, qui est le rapport sur lequel se fonde la reconnaissance iconique des figures (par l'effet de la pression gestaltique). En bref, on voit peu à peu se substituer, dans la peinture de Duchamp, et tout particulièrement dans la repré sentation du corps, la figure sémiotique de l'empreinte à celle de l'icône. Les corps humains sont encore des «icônes», mais ils n'entretiennent plus avec leur environnement le rapport d'une figure avec son fond: en s'enfonçant peu à peu danscefond, la figureen fait surgir la composition matérielle, y suscite la forme d'une empreinte et prépare ainsi la désiconisation radicale qui suivra.

Restent deux autres représentations de nus féminins quen'entoure aucun halo, et qui pourraient donc se présenter comme des figures sur un fond (Le gaz d'éclairage et la chute d'eau, étude préliminaire [ill.14]; et Étant donnés: $1^{\circ}$ la chute d'eau, $2^{\circ}$ le gaz d'éclairage). La comparaison entre les deux, I'une étant une étude préliminaire à l'autre, est particulièrement instructive.

La premièrene peut être vue qu'à traversun trou percé dans une paroi, installant ainsi une profondeur par évidement, sur le modèle dela composition internedel'œil: un orifice, à partir duquel se forme, sur l'enveloppe intérieure, une projection. Cette projection sefait sur un fond qui, de fait, n'en est plus un: des feuilles d'une matière brune sont placées autour du corps ou même sur les membres. Le corps est entouré d'une ligne noire, qui empêche qu'on puisse le voir comme un relief se détachant sur le fond de la surface brune, qui invite au contraire à le voir comme s'il s'enfonçait dans cette matière. Dans cette étude préliminaire, la profondeur se creuse dans l'enveloppe, la projection recule dans la matière même de cette dernièreet commenceà y dessiner son empreinte.

La seconde se présente comme une déchirure dans une toile noire, derrière laquelle on découvre un corps de femme étendu dans un paysage: la vision est incomplète, les parties visibles sont tout juste reconnaissables. O n assiste ici à la même perversion du dispositif de la profondeur: de trou en déchirure et de déchirure en fente, l'œil du spectateur est censé reconnaître, au premier plan de cette masse rose étalée dans I'herbe, dans une forme qui ne semble à première vue qu'une autre vague déchirure, la forme d'une vulve qui lui indique, par contiguïté, la présence d'un corps féminin. Il y a ici, certes, une profondeur, maisc'est déjà celle du creusement, de la fissure ou de la déchirure, et non celle du relief et du modelé.

$D$ ans un cas comme dans l'autre, la figure principale est celle de l'enveloppe, qu'elle soit saisie de l'extérieur comme une forme vide ou de l'intérieur comme une surface de projection des corps en vue d'y former une empreinte. En somme, Duchamp est en train, alors, d'inventer le dispositif de l'empreinte et du moule et d'imaginer la représentation du corps comme une représentation «en creux».

$M$ ais ces quelques observations sur les enveloppes «extérieures» invitent à un commentaire plus général : il est bien clair maintenant que la figure del'empreinten'est pas d'abord érotique ou sexuelle, mais qu'elle rencontre, massivement mais occasionnellement, la thématique sexuelleet érotique. La figure del'empreinteest très exactement le mode sous lequel D uchamp entend non pas représenter (car il le «représente» en partie de manière iconique et figurative) mais signifier le corps humain, comme un jeu d'enveloppes et d'empreintes en abîme: moulages de l'intérieur, enveloppes viscérales qui 
semblent des moulages del'intérieur du corps, corps euxmêmes, enfin, qui semblent soit des enveloppes vides soit des empreintes dans des moules.

Signifier lecorpshumain commeun jeu d'inscriptions et d'empreintes, c'est conférer à sa représentation une toute autre dimension que celle d'une icône. Comme insistejustement $\mathrm{H}$ erman Parret, la peinture de D uchamp fuit le symbolisme, l'allégorie et la métaphore, elle est «indicielle». M ais il faut entendre ici «indice» non pas au sens de la simple métonymie narrative, qui fait que tout événement peut être signalé par la manifestation de ses conséquences, mais au sens de l'inscription durable dans une matière: Duchamp s'efforce de saisir et de conserver dans la matière de la peinture la trace et l'inscription reconnaissable d'un contact éphémère, d'un passage fugace et d'un retour toujours différé; il cherche à imprimer une absence, pourtant si présente dans la forme abandonnée dans l'empreinte, un fantasme, en somme, celui du corps humain.

II ne s'agit plus alors de représenter mais d'inscrire, d'imprimer ce qui ne peut directement être saisi. 0 n voit bien que, du même coup, le processus sémiotique est inscrit dans une perspective phénoménologique: celle de la présenceet des modal ités de la présence. N ous avancions pour commencer, à propos del'interaction entrela choseet l'objet, qu'un basculement des modes d'existence (actuel et réel/ virtuel et potentiel) était requis. N ous pouvons maintenant ajouter que seule la figure, la forme-empreinte, témoigne de la présencede ses divers modes de «remplissement», et aussi de leur identité figurative (comme l'indique le terme de «figure».

\section{Conclusion : empreinte, concept et signature}

Lemouledu visage, ainsi queles moulages qu'on peut en tirer, est doublement une marque d'identité: en tant que visage et en tant qu'empreinte. En tant que visage, il constituele «meilleur échantillon» del'identitécorporelle, et son pouvoir individualisant est, pour des raisons qui restent à explorer, beaucoup plus fort que bien d'autres, plus fréquemment utilisés par Duchamp: sexes, seins, torses, etc. En tant qu'empreinte, il constitueuneidentité stable, qui résiste aux aléas de la chair et du temps et qui supporte l'absence, même définitive.

Lesfemmes peintes par D uchamp n'ont leplus souvent pas de visage, a fortiori quand elles n'ont plus de corps et rien que des viscères. En revanche, les hommes (D octeur D umouchel et lui-même, dans son Autoportrait de 1968 [ill. 12]) ont un visage. Peut-être peut-on retrouver là un principe degénéricitéquel'absencede relationsd'analogie et d'équivalence interdisait. Le corps des femmes est "générique» en ce qu'il est essentiel; celui des hommes, à commencer par celui de l'artiste, est «individuel » parce qu'il est accidentel: la présence ou l'absence des visages est alors à rapprocher de ce que nous disions plus haut du «féminin» et du «masculin »: le «masculin » n'est qu'une des possibilités d'actualisation et de «remplissement» de l'enveloppe intérieure du «féminin » et de son empreinte inscrite sur la toile.

Le visage ne peut pas, chez D uchamp ${ }^{16}$, subir le processus d'excavation et de désincarnation qui mettrait à nu la «machine» et son enveloppe intérieure; aussi son moulage ou sa représentation peinte conservent-ils leur caractère de signature irréductiblement individuelle ( $M$. D umouchel a un nom propre, tout comme l'autoportrait del'artiste, alors que les femmes ne sont que des «vierges» ou des «mariées»). En revanche, l'empreinte du corps féminin sans visage, son enveloppe extérieure ou son empreinte intérieure ont valeur de signature de l'espèce toute entière, ou du moins ici du «genre» féminin en tant que tel. 


\section{N OTES}

1. U. Eco, Kant et l'ornithorynque, Paris, G rasset, 1999.

2. N otamment dansD . Anzieu, LeC orpsdel'œuvre, Paris, Gallimard, 1981.

3. Selon la proposition d'A. Zinna, "L'objet et ses interfaces», dans J. Fontanille et A. Zinna (dir.), Les O bjets au quotidien, Limoges, Pulim (à paraitre).

4. Pour convaincredu rôle du débrayage dansl'empreinte, on peut appeler à la barre des témoins U. Eco: «L'empreinte est un signe en tant qu'elle est fondamentalement une expression qui renvoie à un contenu, et un contenu est toujoursgénéral. LorsqueRobinson découvreuneempreintesur lesable, il nedit pas V endredi est passé par là, mais U ne créature humaine est passée par là» (op. cit., p. 378). D ans le processus d'interprétation de l'empreinte, en une première phase, le contenu est générique: cette généricité implique un débrayage à partir du corps individuel qui a laissé sa trace.

5. Commeil s'agit là seulement du principe destabilisation dela figure, et non de sa capacité à représenter par analogie une autre figure, il vaudrait mieux parler d'hypoicône et réserver le terme d'icône au deuxième cas. Cf. J.-F. Bordron, «Réflexions sur la genèse esthétique du sens», Protée, vol. 26, n०2, 1998, p. 87-104.

6. On conviendra ici de distinguer l'empreinte et le moule de la manière suivante: l'empreinte est le cas générique minimal ; le moulage y ajoute une procédure concertée, programmée et volontaire (un vouloir faireet un savoir faire, en quelquesorte) ; en outre, si l'empreintepeut êtrepartielle, imparfaite et accidentelle, le moulage doit être complet, le plus fidèle possible, etc. O n peut faire un moulage aussi bien à partir d'une empreinte qu'à partir d'un moule, maisil s'agit al orsdelarestitution delaformeabsente, grâceàlaquelle I'objet vient prendre la place de la chose absente, il ne s'agit plus de la figure (empreinte ou moule) qui assure la stabilité et l'identité du couple «chose/ objet».

7. U . Eco répond à une discussion de Bacchini en ces termes: «Bacchini dit que l'empreinte est séparée temporellement de l'objet imprimeur, mais non spatialement, car elleest “ contiguë" à l'objet imprimeur, auquel ellecorrespond point par point. II me semble que l'on confond ici la coprésence temporelle, la contiguïtéspatialeet lerapport decongruence (un rapport purement formel, qui subsiste également dans le cas du masque mortuaire d'une personne disparue depuislongtemps» (op. cit., p. 379). O n peut donner raison à Eco, en ce sens que la contiguïté spatiale ou la coprésence temporelle éphémère entre le corps et l'empreinte laisse place, dans la durée, à une congruence purement morphologique; lepassage del'uneàl'autre reposetoujours sur le débrayage delaformecongruentepar rapport au corpsqui l'a produite, et cedébrayage est à la fois ou indifféremment spatial et/ou temporel.

8. A.J. Greimas et J. Courtés, Sémiotique. Dictionnaire raisonné de la théorie du langage, Paris, H achette, 1979.

9. Ici même, p. 89.

10. Ici même, p. 90.

11. N ous avons identifié, sous forme de dessin sur papier, annotée et commentée par D uchamp, une ébauche de cette figure (dans J. M ink, $M$ arcel D uchamp, Köln, Taschen, 1995, p.61). Cette ébauche, intitulée Cylindre sexe (guêpe) (1934, coll. A. Schwarz, M ilan), est composée d'un cylindreenveloppéd'un cône, auquel lesannotationsattribuent despropriétés de rotation, d'échauffement, de ventilation et de circulation entre un «réservoir » et un «archétype» extérieurs à la figure.

12. Ici même, p. 99.

13. Pour préciser cepoint, qui pourrait nousmener sur lechemin dessignes del'ergonomie, on pourrait comparer le rôle de l'empreinte corporelle sur I'instrument àcelledel'icônedansun pictogramme: sur lepictogrammequi nous interdit de fumer dans les lieux publics, une cigarette allumée est représentée et barrée de rouge, mais cette image ne signifie pas pour autant «ceci n'est pas une cigarette»; il faut donc interpréter le pictogramme dans le sens de l'acte, sur lequel il fait porter une interdiction, et non dans celui de la représentation de l'objet; de la même manière, l'instrument, par I'ergonomie de sa forme, dit bien à quelle partie du corps il s'adresse, mais il faut l'interpréter dans le sens de l'acte qu'il permet d'accomplir, et non comme une représentation en moulage de l'organe.

14. H. Parret interprète le motif du «gaz d'éclairage» comme une représentation des fluides masculins, susceptibles de combler, comme les autres fluides qui les occupent, les organes internesféminins. $M$ ais, dans ce cas, les propriétés d'expansion du gaz en font un bien meilleur candidat au rôle de «moulage interne» que toute autre partie du corps masculin, puisqu'il est susceptible d'adopter strictement la forme de l'«enveloppe» intérieure.

15. D ansl'étudedéjàévoquéeplusieursfois, $H$. Parret évoquela prédilection deD uchamp pour lethèmedel'«infra-mince»: deux domaines, deux figures, deux contraires ne sont séparés que par une pellicule infimeet, emboîtés I'un dans l'autre, ils apparaissent de fait comme des complémentaires. Sous le régime des frontières catégorielles «infra-minces», les oppositions sont donc converties en contiguïtés, en emboîtements inséparables.

16. Latentativesignaléepar $H$. Parret d'un moulageen plâtre, qui s'enfonce dans la joue d'un visage, rend celui-ci, justement, méconnaissable en tant que tel. 